\title{
The Time Constant of the Somatogravic Illusion
}

\author{
B. J. Correia Grácio • K. N. de Winkel • \\ E. L. Groen · M. Wentink • J. E. Bos
}

Received: date / Accepted: date

\begin{abstract}
Humans perceive tilt when experiencing a sustained acceleration. This tilt illusion is commonly referred to as the somatogravic illusion. Although the physiological basis of the illusion seems to be well understood, the dynamic behavior is still subject to discussion. In this study the dynamic behavior of the illusion was modeled and the time constant was measured experimentally. Subjects were exposed to pure centripetal accelerations in the lateral direction and were asked to indicate their tilt percept by means of a joystick. Variable radius centrifugation during constant angular rotation was used to generate three different motion profiles. Results showed that the time constant of the somatogravic illusion is in the order of two seconds contradicting the high time constant found in fixed radius centrifugation studies. The model fit was not improved when using an otolith model sensitive to high frequency accelerations. Apart from the fundamental importance, these results also have practical consequences for the simulation of sustained accelerations in motion simulators.
\end{abstract}

Keywords Somatogravic illusion · Acceleration - Tilt · Motion perception · Gravity $\cdot$ Subjective vertical

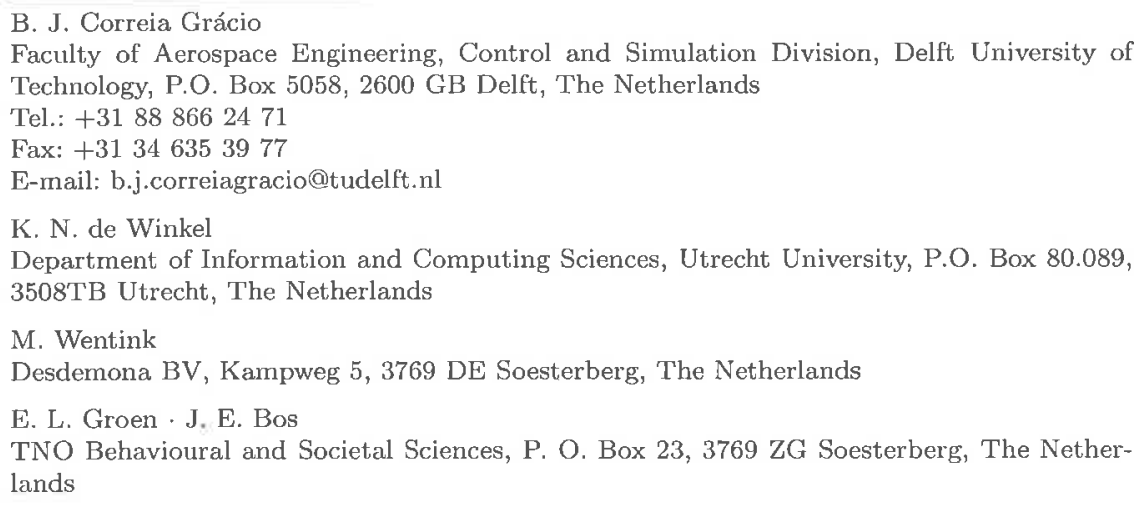




\section{Introduction}

It has been shown that sustained linear accelerations can induce an illusion of whole body tilt (Guedry, 1974; Mayne, 1974; Seidman, Telford, \& Paige, 1998; Clément, Moore, Raphan, \& Cohen, 2001; Merfeld, Zupan, \& Gifford, 2001; Bos \& Bles, 2002; Holly, Vrublevskis, \& Carlson, 2008). This sensory illusion has been commonly referred to as the somatogravic illusion, and originates in the otolith organs of the inner ear because of its incapacity to distinguish between linear acceleration due to self-motion and gravity. In aviation, this illusion has caused fatal accidents. For example, during a catapult launch from an aircraft carrier in poor visual conditions, fighter pilots may misperceive the horizontal take-off acceleration as pitching upward. The tendency to compensate for this pitch-up sensation has caused pilots to crash planes into the ocean (Cohen, Crosbie, \& Blackburn, 1973; Cohen, 1976, 1977). Researchers have shown that the dynamic behavior of this pitch-up sensation may be approximated by a first order low pass filter (Mayne, 1974; Seidman et al., 1998; Bos \& Bles, 2002). Although the physiological basis of the somatogravic illusion seems to be well understood, the main time constant of the illusion (i.e. the time it takes for the tilt percept to reach $63.2 \%$ of its steady stable value) is still subject of discussion (Holly et al., 2008).

Previous studies have been measuring the time constant of the somatogravic illusion by using different methods to generate sustained linear accelerations (Clark \& Graybiel, 1966; Glasauer, 1995; de Graaf et al., 1996; Bos \& Bles, 2002). Clark and Graybiel (1966) studied the somatogravic illusion in a human centrifuge where the linear or centripetal acceleration is generated by eccentric rotation of the subject. In these conditions, they found a time constant of about 20 seconds. Other centrifuge studies on the somatogravic illusion reported time constants of the same order of magnitude (Graybiel \& Brown, 1951; Young \& Meiry, 1968; Guedry, 1974). However, lower time constants were found in studies where sinusoidal linear acceleration was generated on a linear track rather than in a centrifuge (Glasauer, 1995; de Graaf et al., 1996). Under such conditions, de Graaf et al. (1996) observed a time constant of approximately 5 seconds. Based on data from fixed-radius centrifugation and linear sled studies, Bos and Bles (2002) estimated a time constant between 1 and 2.8 seconds. The authors reasoned that the longer time constant observed in centrifuge studies could be due to the angular acceleration of the centrifuge causing stimulation of the semicircular canals. This angular acceleration is required in centrifuges with a fixed-radius to reach supra-natural centripetal accelerations. Indeed, based on theoretical arguments, the cross-coupling between semicircular canals and otolith organs in fixed-radius centrifugation was shown to increase the time constant of the somatogravic illusion to the order of tens of seconds (Bos \& Bles, 2002), depending on the centrifuge angular velocity.

In this study we investigated the time constant of the somatogravic illusion in a centrifuge with variable radius. Using this centrifuge technique, subjects are rotated on-centre up to a constant angular velocity. Due to the high- 
frequency dynamics of the semicircular canals the rotation sensation fades out after tens of seconds of rotating at constant velocity (Guedry, 1974). Then, the subject is translated along the radius to an eccentric position, while maintaining constant angular velocity. This generates a centripetal acceleration in the lateral direction without parasitical stimulation of the semicircular canals. This centrifugation technique has been employed in two prior studies (Seidman et al., 1998; Merfeld et al., 2001). Seidman et al. (1998) created a pitch-tilt illusion by generating a centripetal acceleration in the naso-occipital axis. In their study, the somatogravic illusion was modeled by a transfer function with one pole and one zero in series with a first order low pass filter. It was found that a time constant of 7 seconds for the low pass filter best described their experimental data. Merfeld et al. (2001) compared the roll-tilt illusion induced both by variable-radius and fixed-radius centrifugation. The sensation of tilt changed more rapidly in the experimental conditions using variable-radius centrifugation. However, they did not report any value for the time constant found during the variable-radius centrifugation.

In the current paper we describe a variable-radius centrifuge experiment using the Desdemona research simulator (Bles \& Groen, 2009). The motion profiles induced a clockwise roll-tilt illusiom from the perspective of the subject. To obtain the time constant from the experimental data, we fitted the Mayne equation (Mayne, 1974; Bos \& Bles, 2002), which describes the perceived gravitational vector as a function of the semicircular canals and otolith afferents. We hypothesized the main time constant of the somatogravic illusion to be in the order of seconds; similar to what was found during the linear sled studies of de Graaf et al. (1996) and later complemented by Bos and Bles (2002).

\section{Methods}

\subsection{Subjects}

A total of six subjects (three male; mean age 25 years, $\mathrm{SD}=4$ )) participated in this experiment. All subjects were paid a standard fee and gave their informed consent after general instructions. The institutional Ethical Committee approved all experimental conditions in the study. Subjects were informed about their rights according to the Declaration of Helsinki on ethical principles for medical research involving human subjects. The subjects reported no history of vestibular dysfunction.

\subsection{Motion platform}

The study was conducted at the Desdemona research simulator (Figure 1). The simulator has six degrees-of-freedom (DoF) that allow centrifuge-based motion simulation (Bles \& Groen, 2009). The simulator cabin is mounted in a 
three DoF gimbaled system that permits full rotations in pitch, yaw and roll. The gimbaled system itself is mounted on an eight meter linear track, which is capable of rotating around its central Earth-vertical yaw axis to produce planetary motion. Using the gimbal system, the subjects' naso-occipital axis was perpendicular to the linear sled which generated centripetal acceleration along the subjects' lateral axis. This induced a sensation of lateral self-tilt (roll). To generate the motion profiles, the central yaw axis rotated to a constant angular velocity. To generate the centripetal acceleration, the cabin moved from the center of the linear track to an off-center position. This movement created three different motion profiles.

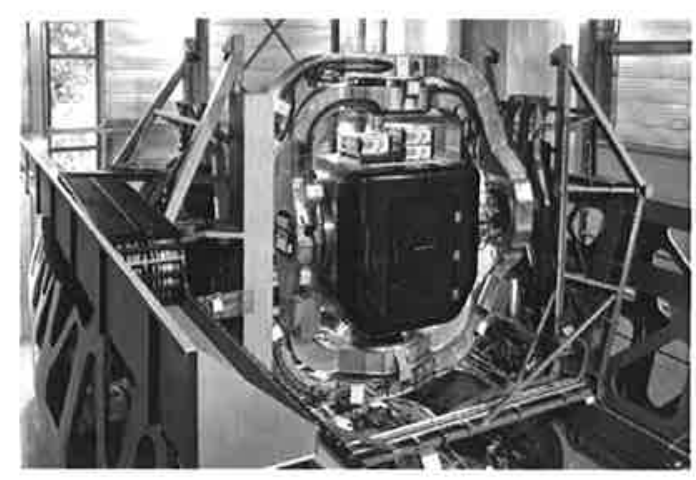

Fig. 1 Desdemona research simulator.

\subsection{Motion profiles}

All motion profiles used in this study had a theoretical centripetal acceleration of $4.1 \mathrm{~m} / \mathrm{s}^{2}$ in the lateral direction causing a (roll) tilt of 22.5 degrees of the specific force away from the gravity vector. To generate such profiles, the centrifuge rotated at a constant angular velocity of $80 \mathrm{deg} / \mathrm{s}$. An acceleration of $5 \mathrm{deg} / \mathrm{s}^{2}$ was initially used to reach this constant angular velocity. During the $5 \mathrm{deg} / \mathrm{s}^{2}$ angular acceleration the centripetal acceleration was zero because the simulator cabin was at the center of rotation. The centripetal acceleration was generated only after the subject reported no perception of yaw-rotation anymore, which generally happened well within 60 seconds. To generate the centripetal acceleration, a raised-cosine velocity profile moved the simulator cabin $2.15 \mathrm{~m}$ outward. This motion profile guaranteed a smooth movement of the simulator actuator over the radius. Equation (1) describes the cabin movement along the centrifuge arm, where $d$ is the final distance of the simulator cabin along the centrifuge arm, $t_{\text {start }}$ is the time at which the simulator cabin starts to move, and $f_{r}$ is the frequency, in $\mathrm{Hz}$, of the raised-cosine leading up to the steady-state acceleration. Using four different frequencies for $f_{r}$ we 
generated four different motion profiles. The frequency values were 0.05, 0.1, and $0.2 \mathrm{~Hz}$.

$R(t)= \begin{cases}0 & , t<t_{\text {start }} \\ -d f_{r}\left(\left(t-t_{\text {start }}\right)-\frac{\sin \left(2 \pi f_{r}\left(t-t_{\text {start }}\right)\right)}{2 \pi f_{r}}\right) & , t_{\text {start }} \leq t<t_{\text {start }}+\frac{1}{f_{r}} \\ -d & , t_{\text {start }}+\frac{1}{f_{r}} \leq t\end{cases}$

The generated centripetal acceleration is given by Equation (2), where $a_{c}$ is the centripetal acceleration, $\omega$ is the angular velocity of the centrifuge arm and $R$ is the distance, as given by Equation (1).

$$
a_{c}(t)=\omega^{2} R(t)
$$

Apart from the lateral centripetal acceleration and gravity, a Coriolis acceleration is at issue, acting on the naso-occipital axis of the subject. Together all linear accelerations compose the gravito-inertial acceleration, or specific force. Equation (3) shows the specific force $(f)$, where $f_{x}$ is the specific force acting on the subjects naso-occipital axis, $f_{y}$ is the specific force acting on the subjects lateral axis, $f_{z}$ is the specific force acting on the subjects' vertical axis and $g$ is the magnitude of the gravitational acceleration $\left(9.81 \mathrm{~m} / \mathrm{s}^{2}\right)$. Figure 2 shows the time histories of the specific force components for the three different motion profiles.

$$
f=\left\{\begin{array}{l}
f_{x}=-2 \omega \dot{R} \\
f_{y}=-\omega^{2} R+\ddot{R} \\
f_{z}=g
\end{array}\right.
$$

\subsection{Joystick}

For measuring the roll-tilt illusion we used a custom-made rod which is not affected by any linear acceleration (i.e, gravity neutral) due to its symmetrical design. A schematic of the joystick is shown in Figure 3. This joystick was fixed to the subject's seat. Subjects were asked to indicate their perceived roll-tilt by rotating the rod in the same direction as their perception. Therefore, for a roll-tilt to the right, subjects had to move the rod also to the right. Joystick rotation was physically limited to rightward rotations from upright onwards. A button next to the gravity neutral rod allowed subjects to indicate when they were ready to proceed to the next experimental trial. 
B. J. Correia Grácio et al.

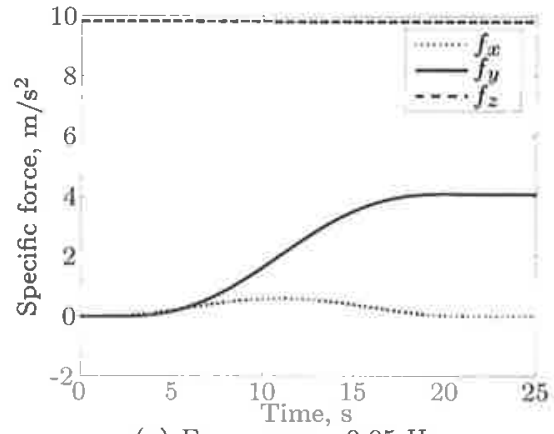

(a) Frequency $=0.05 \mathrm{~Hz}$

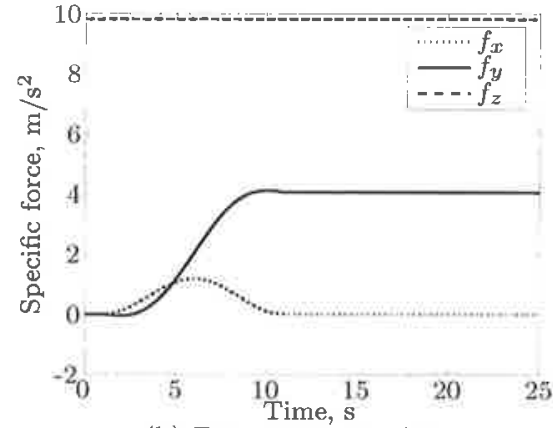

(b) Frequency $=0.1 \mathrm{~Hz}$

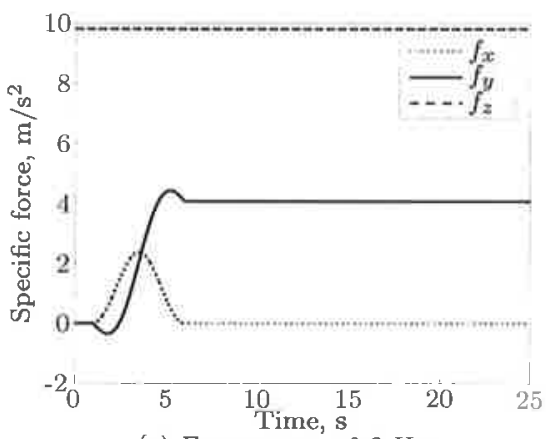

(c) Frequency $=0.2 \mathrm{~Hz}$

Fig. 2 Specific force components acting on the subjects for the four different motion profiles.

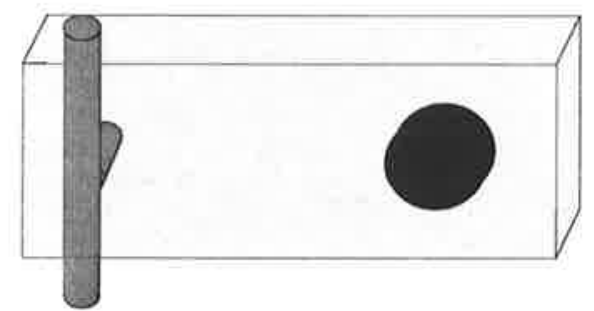

Fig. 3 Schematic of the joystick used to measure the roll-tilt illusion. The rod had a diameter of $1.5 \mathrm{~cm}$ and a length of $13.5 \mathrm{~cm}$. Both the rod and the knob were operated using the right hand.

\subsection{Experimental design}

Although the experiment consisted of two additional conditions including vision, in this paper we only describe the experimental conditions without a visual stimulus. Each subject was measured seven times for each of the three different motion profiles. This leads to a total of 21 experimental trials for each 
of the six subjects. The order in which subjects performed the experimental conditions and its repetitions was randomized.

\subsection{Procedure and subjects' instructions}

Subjects were seated in the simulator cabin and secured by a five-point safety belt. Subjects were instructed not to move their head during the experiment. A headrest provided head support. These measures were intended to minimize coriolis and keep their head aligned with the Earth vertical. Subjects wore a headset on which white noise was presented to mask actuator sound. The headset also allowed for communication between the subject and the experimenter. All experimental trials were performed with eyes closed in a dark cabin. The experimental trials started by rotating the centrifuge arm until a constant angular velocity was reached. During this rotation the simulator cabin remained in the center of the centrifuge arm. Subjects communicated to the experimenter when the perception of yaw rotation had disappeared. With an additional delay of six seconds, subjects then initiated the lateral motion by pressing the knob next to the joystick. The start of the cabin movement was signaled to subjects by a, 1.5 seconds sound cue via the headset. While the cabin was moving outward, subjects constantly indicated their subjective roll-tilt perception by keeping the rod aligned with the perceived roll angle. Subjects communicated to the experimenter when a steady tilt illusion was obtained. After a verbal signal from the experimenter, subjects pressed the knob again and the cabin returned to the center position. When the simulator cabin was back at the center position, 6 seconds passed before automatically proceeding to the next experimental trial. This procedure was repeated until all the 21 experimental trials where completed.

\subsection{Data analysis}

\subsubsection{The Mayne equation}

The input of the otoliths is the three-dimensional specific force $(\vec{f})$, as defined in Equation 4 where $\vec{a}$ is the acceleration vector due to motion and $\vec{g}$ is the acceleration vector due to gravity.

$$
\vec{f}=\vec{a}+\vec{g}
$$

From Equation (4) it follows that the otoliths alone cannot discriminate between accelerations due to self-motion and accelerations due to gravity. However, the Central Nervous System (CNS) seems to be able to estimate both components (Mayne, 1974; Bos \& Bles, 2002), otherwise humans would perceive acceleration due to gravity as linear movement which induce a constant percept of moving upwards. Mathematical models have been proposed to explain how the CNS may distinguish between the two different accelerations. 
An elegant and simple model was proposed by Mayne (1974). Although the original equations were described for two dimensions, Bos and Bles (2002) turned these to one 3D coupled equation. This equation is described by Equation (5), where $f_{\text {oto }}$ is the specific force signal given by the otolith afferents, $\omega_{s c c}$ is the angular velocity signal given by the semicircular-canal afferents, $\tau$ is the time constant of low-pass filter operating on the otolith afferents, and $\tilde{g}$ is the estimation of the acceleration due to gravity as taken by the CNS.

$$
\frac{d \tilde{g}}{d t}=\frac{1}{\tau}\left(f_{o t o}-\tilde{g}\right)-\omega_{s c c} \times \tilde{g}
$$

Equation (5) takes the combined outputs from the otolith organs and semicircular canals to produce an estimate of acceleration due to gravity $(\tilde{g})$ and the estimation of acceleration due to $\operatorname{motion}\left(\tilde{a}=f_{\text {oto }}-\tilde{g}\right)$. Bos and Bles (2002) showed that the Mayne equation could predict a large number of motion/orientation illusions, in particular the somatogravic illusion.

Since our method of inducing the somatogravic illusion did not involve stimulation of the semicircular canals, Equation (5) can be simplified into Equation (6). Equation (6) is equivalent to a first order low-pass filter with a time constant equal to $\tau$.

$$
\frac{d \tilde{g}}{d t}=\frac{1}{\tau}\left(f_{o t o}-\tilde{g}\right) \Leftrightarrow \tilde{g}=\frac{1}{\tau s+1} f_{o t o}
$$

We tested two different otolyth functions in this study; one is commonly used in literature (Merfeld, Young, Oman, \& Shelhamer, 1993; Bos \& Bles, 2002) and is defined by a unit gain whereas the other has dynamics as described by Hosman (1996). Therefore, we calculated the time constant of the somatogravic illusion based on Equation (7) and (8), where Equation (7) uses the unit gain otolith model and Equation (8) uses the otolith model described by Hosman (1996).

$$
\begin{gathered}
f_{\text {oto }}=f \Rightarrow \\
\tilde{g}(s)=\frac{1}{\tau s+1} f \\
f_{\text {oto }}=\frac{(s+1)}{(0.5 s+1)(0.016 s+1)} f \Rightarrow \\
\tilde{g}(s)=\frac{(s+1)}{(\tau s+1)(0.5 s+1)(0.016 s+1)} f
\end{gathered}
$$

In terms of model fit, the otolith model described by Hosman should have a better fit since the extra dynamics could allow the fitting algorithm to predict any high frequency dynamics shown in the measured signal. However, these extra dynamics given by the otolith model were fixed to values suggested by the literature and could not be adjusted by the fitting procedure. Therefore, the fitting procedure used the same number of parameters for both models. 


\subsubsection{Processing of joystick data}

The joystick raw data was sampled at $25 \mathrm{~Hz}$. A second order low-pass filter with a cut-off frequency of $4 \mathrm{~Hz}$ was used to filter the joystick-sampled data. The filter $4 \mathrm{~Hz}$ cut-off frequency was well above the important frequencies of the motion profiles. We used a zero-phase forward and reverse digital filtering technique to ensure no phase distortion of the filtered responses. The data of each run was trimmed between the button press to start the cabin movement and the button press that brought the cabin back to the centre of the simulator's planetary arm. Therefore, the higher frequency conditions had fewer data points, due to a faster movement in time, than the lower frequency conditions. The predicted response of Equations (7) and (8) are in gravity components and therefore, not comparable with the roll angle measured by the joystick. Thus, Equation (9) transforms the gravity components to the roll angle predicted to be perceived by humans. In Equation (9), $\tilde{g}_{y}$ and $\tilde{g}_{z}$ are the estimated acceleration due to gravity in the lateral and vertical direction, respectively; and $K$ is the perceived magnitude.

$$
\tilde{\phi}=\tan ^{-1}\left(\frac{\tilde{g}_{y}}{\tilde{g}_{z}}\right) K
$$

A least squares minimization procedure was used to estimate the two parameters of interest, the time constant and the perceived magnitude. The algorithm calculates the value of the parameters that yield the smallest error between the predicted response and the measured data. A fit was obtained for all experimental trials, which led to a total of 21 model fits. For each fit we used the Variance Accounted For (VAF) to evaluate the quality of the fit. The VAF is given by Equation (10), where $u$ is the recorded data and $u_{m}$ is the model clata. A VAF of $100 \%$ means that the measured data and the data generated by the mathematical model are equal. A Repeated Measures ANOVA was used to test if the parameters differed for the different levels of the independent variable.

$$
V A F=\left(1-\frac{\sum\left(u-u_{m}\right)^{2}}{\sum u^{2}}\right)
$$

\section{Results}

Figure 4 shows the mean joystick responses and their standard deviation, combined over six subjects. Each panel represents one of the three different motion frequencies. The roll response for each subject was obtained by averaging over the seven repetitions. Figure 4 also shows the true roll tilt of the specific force in relation to the subjects' spinal axis. The graphs show that the perceived tilt lags in relation to the true tilt of the specific force vector. After a few seconds the roll response reaches its steady state value, close to the true tilt. 


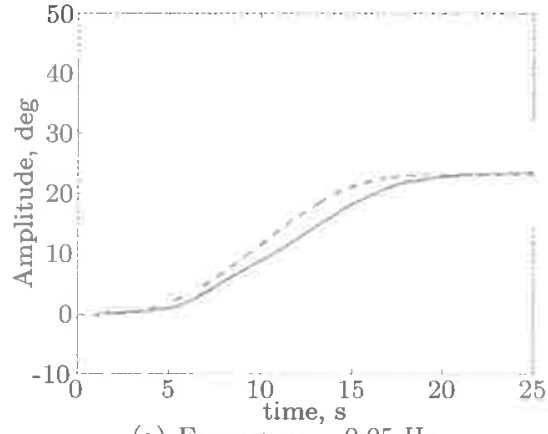

(a) Frequency $=0.05 \mathrm{~Hz}$

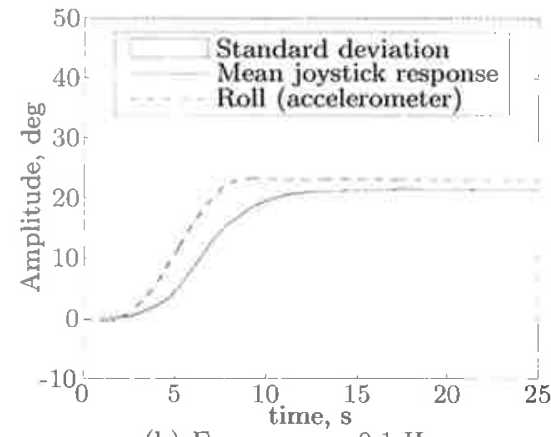

(b) Frequency $=0.1 \mathrm{~Hz}$

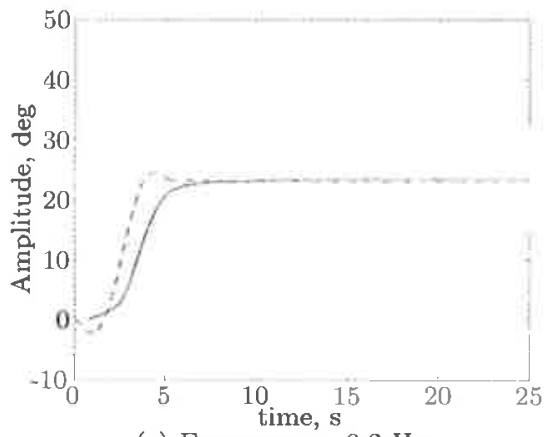

(c) Frequency $=0.2 \mathrm{~Hz}$

Fig. 4 Mean joystick response between the six subjects for the three different motion profiles. The shaded area represents the standard deviation.

Figure 5 shows that the overall VAF values for the fits were high (above $99 \%$ ), meaning that both models were able to accurately describe the measured data. There were no significant differences between the VAFs obtained for the two models. The different frequencies also had no effect on the VAF. The mean VAF was $99.54 \%$.

Figure 5 also shows the mean of the estimated parameters (gain and time constant) across subjects for the two tested otolith models. The results show that the obtained time constants for both models are in the order of seconds. An ANCOVA showed a main effect both of the frequency ( $\mathrm{F}=16.29, p=0.000)$ and otolith model $(\mathrm{F}=15.93, p=0.000)$ on the time constant value. The average values for the unit gain otolith model were 2.04, 1.75, and 1.30 seconds for the $0.05,0.1$, and $0.2 \mathrm{~Hz}$ frequencies, respectively. For the model with otolith dynamics, the average time constants were $2.60,2.36$, and 1.90 seconds for the conditions with $0.05,0.1$, and $0.2 \mathrm{~Hz}$, respectively.

The ANCOVA showed no effects of the otolith model and frequency on the perceived amplitude gain. Figure 5(c) shows that the amplitude gain was around one for all conditions. 


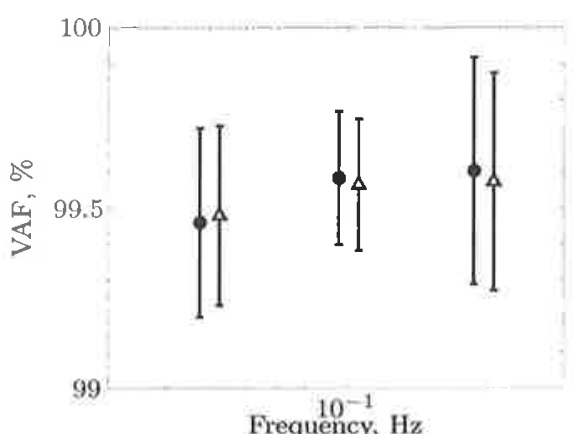

(a) VAF

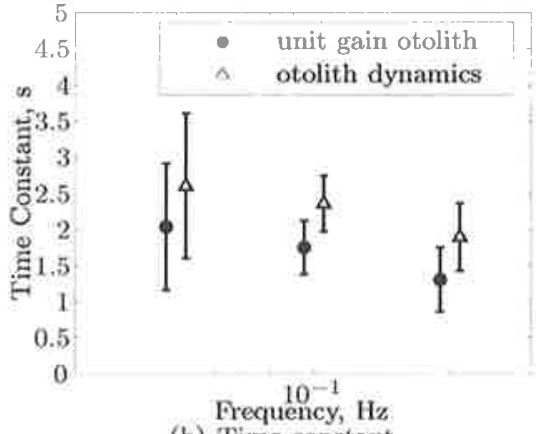

(b) Time constant

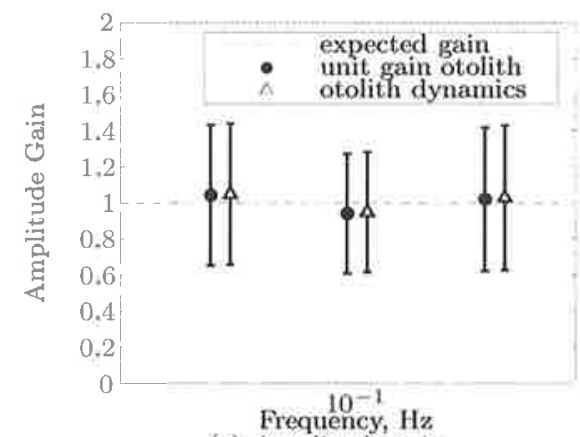

(c) Amplitude gain

Fig. 5 Parameters obtained from the Least squares fitting procedure for both tested models. In the plots we show the mean parameters. The vertical lines represent the $95 \%$ confidence intervals. The dashed line in the amplitude gain and offset parameter plots represents the value that the Mayne equation has in the literature for these parameters. The mean VAFs and their SEMs also shown in this figure.

\section{Discussion}

In this study we investigated the time constant of the somatogravic illusion without being confounded by semicircular canal stimulation. This was achieved by pre-rotating the cabin on the centrifuge axis to constant angular velocity, and subsequently moving the cabin to an excentric position. We found a time constant on the order of seconds, rather than tens of seconds, as often found in studies using fixed-radius centrifugation (e.g., (Graybiel \& Brown, 1951; Clark \& Graybiel, 1966; Young \& Meiry, 1968; Guedry, 1974)). Therefore, our results support the hypothesis that the angular acceleration inherent to fixedradius centrifugation may interfere with the pure otolith-induced somatogravic illusion, as suggested in other studies (Seidman et al., 1998; Merfeld et al., 2001; Bos \& Bles, 2002). Bos and Bles (2002) showed this theoretically using Equation (5). 
The estimated time constant of the somatogravic illusion in this experiment was around 2 seconds, comparable to the values found by Bos and Bles (Bos $\&$ Bles, 2002). In their study they found a time constant for Equation (5) that ranged between 1 and 2.8 seconds. This time constant was measured from experimental conditions where the motion profiles were generated by a linear sled, guaranteeing that angular motion was not present. Despite the different motion profiles between the two studies, the value of the time constant is very similar. Bos and Bles (2002) also used the Mayne equation to estimate the time constant values. Seidman et al. (1998) measured a slightly higher time constant. They found a time constant of 7 seconds for motion profiles generated using variable-radius centrifugation. However, this 7 seconds time constant chosen for the low pass filter in their tilt-illusion model could have been affected by a transfer function that preceded the low pass filter. This transfer function was added in their model for adaptation purposes. We showed that extra linear dynamics, like the otolith transfer function, could increase the time constant of the low pass filter. Differences between their motion profile and the one used in this study may alternatively explain the different values found for the time constant. The motion profiles differed in direction, amplitude and frequency. The human ability to detect linear motion was found to depend on the direction of motion (Zaichik, Rodchenko, Rufov, Yashin, \& White, 1999; Heerspink et al., 2005), therefore it is reasonable to consider an effect of the degree of freedom on the time constant of the somatogravic illusion.

\subsection{The Mayne equation}

The main time constant of the somatogravic illusion was obtained by fitting the Mayne equation (Mayne, 1974; Bos \& Bles, 2002) to the measured experimental data. We considered two different otolith dynamics when fitting the data: the simplified unit gain dynamics used in some studies (Merfeld et al., 1993; Bos \& Bles, 2002) and the dynamic model fitted by Hosman (Hosman, 1996). For the three different measured frequencies, the mean VAF of the fitted Mayne equations were above $99 \%$. This means that the used models were able to predict on average more than $99 \%$ of the subjects responses.

When considering the otolith physiology, it is reasonable to consider an otolith model with dynamics (Hosman, 1996). In this study, the VAF results showed that the extra dynamics introduced in the otolith model did not improve the fit. These extra dynamics are used to model high-frequency behavior found in humans and animals (Fernandez \& Goldberg, 1976; Benson, Spencer, \& Stott, 1986; Hosman, 1996; Grant \& Haycock, 2008; Soyka, Robuffo Giordano, Beykirch, \& Bülthoff, 2011). However, the motion profiles used in this study had mainly low-frequency content since they were sustained accelerations, which might explain why the extra dynamics did not improve the model fit. The obtained time constants are about 0.6 seconds lower for the unit gain model. This results was influenced by the fitting procedure. Although the obtained time constants are different, the properties of Equations (7) and (8) in 
the frequency domain are very similar in the vicinity of the motion profiles frequencies ( 0 to $0.2 \mathrm{~Hz}$ ). The model of Equation (8) needs time constants 0.6 seconds higher to compensate for the increased gain introduced by the extra dynamics.

The results, however, show that the used models are not yet able to completely describe the somatogravic illusion. This is due to the frequency dependency of the time constant. The time constant of the model decreased when the frequency of the motion profile increased. This model would be able to describe the roll-tilt illusion if the time constant would be the same for the different tested frequencies. However, it seems that the time constant is updated for motion profiles with higher frequency content. Therefore, it is necessary to include a mechanism to deal with the update of the time-constant value. The data used in this study is not enough to estimate this mechanism since only six subjects participated in the study, the amplitude was fixed for all motion profiles, and the tested frequencies were low. The frequency range should be more than one decade to better understand the model structure in the frequency domain. In the authors' opinion, a solution to deal with this frequency dependency might be achieved by assuming an internal model, similar to what was done in previous studies (Merfeld et al., 1993; Bos \& Bles, 2002).

\subsection{Practical consequences}

The modeling of the somatogravic illusion is crucial for the simulation field where linear accelerations have to be scaled down and are of limited duration due to the physical limitations of motion simulators. Currently, techniques like tilt coordination (Reid \& Nahon, 1985) use the gravity vector to simulate the total specific force vector. In this technique, researchers tilt the simulation cabin to match the direction of the gravity vector with the direction of the total specific force vector. A rate limiter is used to ensure that the cabin rotations are not perceived by the pilot. However, this rate limiter may create delays perceived by the pilot, which may compromise the simulation realism. Instead of trying to create an unperceived rotation, the technique we propose assumes that humans perceive tilt when subject to linear acceleration and tries to use that knowledge to induce the same tilt perception by tilting the simulator platform without the use of any rate limiter. Therefore, improvements in this self-motion perception model, like including an internal model to deal with the frequency dependency, would directly lead to improvements in motion simulation.

\section{Conclusion}

In this study we used variable-radius centrifugation to study the time constant of the somatogravic illusion. Our results showed that this time constant is in the order of two seconds for lateral accelerations. This result seems congruent 
with other studies where linear acceleration was isolated from angular motion by means of a sinusoidal motion profile.

Although the Mayne equation accurately fitted the measured data, it cannot explain the frequency dependency of the time constant. Introducing highfrequency dynamics in the model did not improve the model fit neither explained the frequency dependency. Therefore, this model needs to be augmented with a mechanism to deal with the frequency dependency of the time constant. Implementing an internal model in the Mayne equation could be a solution to deal with the frequency dependency.

In addition to confirming that the time constant of the somatogravic illusion is in the order of seconds, this study showed that this time constant is dependent of the motion profile frequency content. This study improved not only the fundamental knowledge regarding the somatogravic illusion, but also suggested improvements to the self-motion perception models which ultimately will improve applications using these models, like motion cueing research.

\section{References}

Benson, A. J., Spencer, M. B., \& Stott, J. R. R. (1986). Thresholds for the Detection of the Direction of Whole-Body, Linear Movement in the Horizontal Plane. Aviation, Space, and Environmental Medicine, 57(11), 1088-1096.

Bles, W., \& Groen, E. L. (2009). The DESDEMONA Motion Facility: Applications for Space Research. Microgravity Science and Technology, 21, 281-286.

Bos, J., \& Bles, W. (2002). Theoretical considerations on canal-otolith interaction and an observer model. Biol. Cybern., 86, 191-207.

Clark, B., \& Graybiel, A. (1966). Factors Contributing to the Delay in the Perception of the Oculogravic Illusion. The American Journal of Psychology, 79(3), 377-388.

Clément, G., Moore, S. T., Raphan, T., \& Cohen, B. (2001). Perception of Tilt (Somatogravic Illusion) in Response to Sustained Linear Acceleration during Space Flight. Exp Brain Res, 138(4), 410-418.

Cohen, M. M. (1976). Disorienting Effects of Aircraft Catapult Launchings II. Visual and Postural Contributions. Aviation, Space, and Environmental Medicine, 47(1), 39-41.

Cohen, M. M. (1977). Disorienting Effects of Aircraft Catapult Launchings III. Cockpit Displays and Piloting Performance. Aviation, Space, and Environmental Medicine, 48(9), 797-804.

Cohen, M. M., Crosbie, R. J., \& Blackburn, L. H. (1973). Disorienting Effects of Aircraft Catapult Launchings. Aviation, Space, and Environmental Medicine, 44(1), 37-39.

de Graaf, B., Bos, J. E., Tielemans, W., Rameckers, F., Rupert, A. H., \& Guedry, F. E. (1996). Otolith Contribution to Ocular Torsion and Spa- 
tial Orientation During Acceleration (Technical Memorandum No. 96-3). Pensacola, Fa: Naval Aerospace Medical Research Laboratory.

Fernandez, C., \& Goldberg, J. M. (1976). Physiology of Peripheral Neurons Innervating Otolith Organs of the Squirrel Monkey. III. Response Dynamics. Journal of Neurophysiology, 39(5), 996-1008.

Glasauer, S. (1995). Linear Acceleration Perception: Frequency Dependence of the Hilltop Illusion. Acta Otolaryngol Suppl, 520, 37-40.

Grant, P. R., \& Haycock, B. (2008). Effect of Jerk and Acceleration on the Perception of Motion Strength. Journal of Aircraft, 45(4), 1190 - 1197.

Graybiel, A., \& Brown, R. H. (1951). The Delay in Visual Reorientation Following Exposure to a Change in Direction of Resultant Force on a Human Centrifuge. Journal of General Psychology, 45(2), 143-150.

Guedry, F. E. (1974). Psychophysics of Vestibular Sensation. In H. H. Kornhuber (Ed.), Handbook of Sensory Physiology, vol VI. Vestibular System Part 2: Psychophysics Applied Aspects and General Interpretations (p. 3-154). Springer-Verlag Berlin Heidelberg New York.

Heerspink, H. M., Berkouwer, W. R., Stroosma, O., van Paassen, M. M., Mulder, M., \& Mulder, J. A. (2005). Evaluation of Vestibular Thresholds for Motion Detection in the SIMONA Research Simulator. In AIAA Modeling and Simulation Technologies Conference and Exhibit. San Francisco, CA.

Holly, J. E., Vrublevskis, A., \& Carlson, L. E. (2008). Whole-Motion Model of Perception During Forward- and Backward-Facing Centrifuge Runs. Journal of Vestibular Research, 18(4), 171-186.

Hosman, R. J. A. W. (1996). Pilot's perception and control of aircraft motions. Unpublished doctoral dissertation, Faculty of Aerospace Engineering, Delft University of Technology.

Mayne, R. (1974). A Systems Concept of the Vestibular Organs. In H. H. Kornhuber (Ed.), Handbook of Sensory Physiology, vol VI. Vestibular System Part 2: Psychophysics Applied Aspects and General Interpretations (p. 493-580). Springer-Verlag Berlin Heidelberg New York.

Merfeld, D. M., Young, L. R., Oman, C. M., \& Shelhamer, M. J. (1993). A Multidimensional Model of the Effect of Gravity on the Spatial Orientation of the Monkey. Journal of Vestibular Research, 3, 141-161.

Merfeld, D. M., Zupan, L. H., \& Gifford, C. A. (2001). Neural Processing of Gravito-Inertial Cues in Humans. II. Influence of the Semicircular Canals During Eccentric Rotation. Journal of Neurophysiology, 85, 1648-1660.

Reid, L. D., \& Nahon, M. A. (1985). Flight simulation motion-base drive algorithms: part 1 - developing and testing the equations (Tech. Rep. No. 296). Institute for Aerospace Studies, University of Toronto.

Seidman, S. H., Telford, L., \& Paige, G. D. (1998). Tilt perception during dynamic linear acceleration. Exp Brain Res, 119(3), 307-34.

Soyka, F., Robuffo Giordano, P., Beykirch, K. A., \& Bülthoff, H. H. (2011). Predicting Direction Detection Thresholds for Arbitrary Translational Acceleration Profiles in the Horizontal Plane. Exp Brain Res, 209(1), 97-107. 
Young, L. R., \& Meiry, J. L. (1968). A Revised Dynamic Otolith Model. Aerospace Medicine, 39(6), 606-608.

Zaichik, L. E., Rodchenko, V., Rufov, I. V., Yashin, Y. P., \& White, A. D. (1999). Acceleration Perception. In AIAA Modeling and Simulation Technologies Conference and Exhibit (p. 512-520). Portland, OR. 